\title{
From Shakespeare to Modern Ages
}

La letteratura per l'infanzia nell'era dei social media Dott. Sayyid KOTB AMIN KOTB

La presente tesi ha l'obiettivo di analizzare la situazione della letteratura italiana per l'infanzia nell'era dei social media, nell'epoca della rivoluzione digitale, cioè in un'epoca in cui tutti gli aspetti della vita quotidiana sono condizionati dall'uso e dallo sviluppo continuo delle moderne tecnologie, e in cui tali tecnologie influiscono ampiamente sullo scarso numero di libri letti da bambini e ragazzi. Inoltre la presente tesi mira ad analizzare gli aspetti positivi e negativi dell'eccessiva dipendenza dai cosiddetti digital devices e le potenzialità che essi offrirebbero al settore pedagogico sia nelle scuole che nelle università.

elementi:

La mia tesi si basa in modo particolare su alcuni

- Definizione, importanza ed esemplari della cosiddetta letteratura per l'infanzia;

- la rivoluzione digitale e la letteratura digitale e / o elettronica;

- i nuovi approcci della didattica della letteratura destinati particolarmente ai ragazzi e adattati alle nuove tendenze culturali;

-l'influenza dei social media sulle nuove modalità e sui nuovi metodi adottati per la diffusione e la promozione della letteratura italiana per l'infanzia.

-Queste tecnologie hanno tutte un effetto negativo o alcune possono stimolare curiosità e partecipazione?

-Si vuole con questo intervento raggiungere la risposta alla domanda assai cruciale: le nuove tecnologie uccideranno il libro ossia lo salveranno?

La letteratura per l'infanzia (o per ragazzi) svolge un ruolo importante nella cultura contemporanea in quanto il libro 


\section{Dahlia Kashmiry, PhD}

per i ragazzi non si ritiene più solo uno strumento didattico, ma una vera e propria opera letteraria: da un lato essa costituisce uno dei primi strumenti espressivi che sono alla base della comprensione del mondo da parte dei ragazzi. In effetti le storielle raccontate si ritengono una fonte preziosa di immagini, parole, atteggiamenti e situazioni importanti per fare esperienza. Dall'altro lato, la fase dell'infanzia è quella in cui i bambini, anche se inconsciamente, sono più ricettivi e apprendono più facilmente le informazioni, in quanto il loro carattere è molto più duttile rispetto a quello di un adulto.

Negli ultimi decenni la letteratura per l'infanzia in quanto campo di studio ha acquisito una maggiore centralità tanto che essa rappresenta una parte immancabile nei corsi universitari e nel lavoro di numerosi centri di ricerca in tutto il mondo. Il maggiore interessamento dimostrato dal mondo accademico ha portato alcune università ad istituire dipartimenti, biblioteche oppure centri specializzati proprio nello studio della letteratura per l'infanzia: a tal proposito si possono annoverare fra i principali centri: 1'“International Youth Library" di Monaco in Germania, il "National Centre for Research in Children's Literature" di Londra e 1"'Homerton Research Centre for Children's Literature" di Cambridge nel Regno Unito, 1"'International Institute for Children's Literature" di Osaka in Giappone, il "Centre national de la littérature pour la jeunesse" di Parigi in Francia, lo "Svenska Barnboks Institutet" [Istituto svedese per libri per bambini] di Stoccolma in Svezia, e la "Biblioteca Salaborsa Ragazzi" di Bologna in Italia.

L'espressione letteratura per l'infanzia si riferisce spesso a tutte le pubblicazioni destinate ai giovani lettori, dai bambini più piccoli che ancora non sanno leggere, ai ragazzi e adolescenti: oggi esiste una grande varietà di pubblicazioni, dai romanzi classici agli albi illustrati, dal formato cartaceo a quello digitale. La letteratura per l'infanzia è un genere particolare e

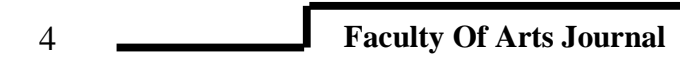




\section{From Shakespeare to Modern Ages}

complesso, ed è alquanto difficile e complesso raggiungere una definizione univoca e ben precisa della letteratura per l'infanzia e delinearne i confini: sia per la vastità del campo e il suo carattere inconsistente sia per la peculiarità del pubblico cui è destinata. Una domanda centrale nell'ambito accademico è se la letteratura infantile debba essere definita come le opere scritte e pubblicate per i bambini, oppure come le opere lette dai bambini o per i bambini ad alta voce.

Al di fuori del contesto accademico, l'espressione non appare particolarmente problematica: con "letteratura per bambini", espressione di uso più comune, ad esempio nei giornali, nei media, ossia in ambito scolastico, si fa usualmente riferimento a tutto quel materiale scritto, pubblicato da case editrici specializzate in opere dedicate a giovani lettori, collocato in sezioni dedicate nelle librerie e nelle biblioteche, per essere poi letto dai bambini e dai ragazzi ${ }^{1}$. In ambito accademico, invece, riuscire a fornire una definizione esauriente rappresenta uno dei nodi centrali, nonché una delle questioni essenziali più complesse affrontate dagli studiosi delle svariate discipline legate al campo della letteratura per l'infanzia.

Il primo nodo centrale riguarda proprio la denominazione stessa della letteratura per bambini. Nella lingua inglese, per esempio, il genitivo dell'espressione "children's literature" [dei bambini] non indica chiaramente quali bambini siano presi in considerazione; in altre parole, non risulta comprensibile se si tratti di personaggi-bambini dei testi, di lettori-bambini oppure di lettori reali o impliciti della narrazione ${ }^{2}$. Del resto, sul livello concettuale, parlando con il termine "infanzia" di una struttura sociale determinata da certe condizioni socio-economiche, in culture diverse e in differenti

\footnotetext{
${ }^{1}$ Cfr. Reynolds, Kimberely, Children's Literature: a Very Short Introduction, Oxford University Press, Oxford 2011, p. 1.

${ }^{2}$ Cfr. Grenby, Matthew O. e Reynolds Kimberely (ed.), Children's Literature Studies: a Research Handbook, Palgrave Macmillan. V. 4, Basingstoke 2011, p. 4.
} 


\section{Dahlia Kashmiry, PhD}

momenti storici, l'infanzia non può essere definita, perché la definizione implica un atto logico che riguarda proprio l'esattezza dei confini anagrafici dell'infanzia, mentre l'infanzia va plausibilmente al di là di ogni logica e razionalità.

E non solo. Il termine stesso di "letteratura" in "letteratura per l'infanzia" suscita non meno discussioni: nel mondo accademico si dibatte costantemente su cosa sia realmente la letteratura per l'infanzia, su quali siano le opere che la costituiscono, oppure sull'effettiva esistenza di caratteristiche comuni alla maggioranza dei libri per bambini e per ragazzi, in quanto i confini della letteratura per l'infanzia stessa, intesa come genere, sono poco chiari. Il campo della letteratura per l'infanzia sembra dunque impossibile da studiare a causa della sua notevole vastità, perciò questa letteratura in fin di conti è molto complessa per essere definita in modo univoco.

Definire comunque la letteratura per l'infanzia ricorrendo a delle definizioni pragmatiche, come per esempio considerarla in maniera più ampia, come "qualsiasi libro letto dai bambini", non ha alcun valore pratico, in quanto una tale definizione eccessivamente ampia nonché generica, porterebbe possibilmente a racchiudere qualsiasi tipologia di testo, dai giornali alle opere teatrali. D'altra parte, una definizione che tenga conto esclusivamente di ciò che le case editrici classificano come libri per bambini e per ragazzi è altrettanto discutibile per la sua limitatezza. Ogni editore potrebbe avere criteri propri per decidere quali libri indirizzare a un pubblico di lettori bambini o ragazzi. Di conseguenza, lo stesso titolo non sarà classificato necessariamente allo stesso modo da editori diversi.

Altre definizioni ${ }^{3}$ di questo complesso campo di ricerca partono, invece, dalla descrizione del destinatario della letteratura per l'infanzia, cioè dalla prospettiva del lettore

3 Cfr. Nodelman Perry, Illustration and Picture Books. in Hunt, Peter, "Children's Literature". In P. Nel e L. Paul (ed.). Keywords for Children's Literature, New York University Press, New York 2011, pp. 42-47.

Faculty Of Arts Journal 
From Shakespeare to Modern Ages

piuttosto dal punto di vista della premeditazione degli autori: un "libro per l'infanzia" è una qualsiasi pubblicazione scritta che abbia nel titolo o da qualche altra parte un'evidente allusione al fatto che è dedicato ai bambini o ai giovani lettori. In altre parole, se l'autore originale destina la propria opera soltanto per la lettura da parte dei bambini, allora siamo di fronte a un libro per l'infanzia. Tuttavia, è un fatto frequente che la letteratura scritta per bambini e ragazzi venga spesso letta anche dagli adulti ${ }^{4}$. Il pubblico molteplice è una delle caratteristiche della letteratura per l'infanzia. In alcuni casi ciò richiede che le opere esistano su due pubblicazioni diverse; una semplice per il lettore giovane, e una più complicata per l'adulto.

Non basandosi comunque sull'età del destinatario, le storie accompagnano da sempre l'umanità, da prima ancora dell'avvento della scrittura, cioè quando erano ancora tramandate oralmente. Non esistevano storie specificamente dedicate all'infanzia, poiché non c'era una separazione netta tra mondo adulto e mondo bambino: le fiabe ne sono un esemplare in quanto esse erano il rito serale per cui avveniva la trasmissione del mondo da una generazione all'altra. Le categorie principali della letteratura per l'infanzia, secondo lo studioso Nodelman ${ }^{5}$, sono: la poesia, i libri illustrati, le favole e fiabe, la letteratura saggistica, e la narrativa. Il primo vero esempio di libro precisamente indirizzato ai bambini-e quindi dell'avvio della letteratura per l'infanzia - è la raccolta dei fratelli Grimm, che uscì per la prima volta nel 1812 e che Jacob Ludwig Grimm e Wilhelm Karl Grimm nell'arco di un cinquantennio sottoposero a continue revisioni, per adattare i contenuti a volte troppo forti delle storie ai bisogni educativi dei

\footnotetext{
${ }^{4}$ Cfr. Beckett, Sandra, Crossover fiction : global and historical perspectives, Children's literature and culture, Routledge, New York, 2009, p. 185.

${ }^{5}$ Cfr. Nodelman, Perry, The pleasures of children's literature, Longman, New York 1992, p. 190.
} 
bambini. La raccolta del Basile, del 1620, Lo cunto de li cunti "ovvero lo trattenemiento de peccerille", si diffuse soltanto in ambienti eminentemente colti e letterari e non specificamente presso un pubblico infantile.

La nascita e l'evoluzione della letteratura per l'infanzia, con le sue alterne vicende e fortune, con i suoi momenti di sviluppo e di crisi, ha condizionato profondamente il rapporto tra bambino e libro, che nel Novecento, in particolare a partire dagli anni Settanta, con la nascita di case editrici specializzate e all'avanguardia e con l'incremento degli studi non solo sulla fiaba, ma anche su quella nuova forma di libro che è l'albo illustrato, ha raggiunto dei livelli mai prima conseguiti. Con la diffusione dell'albo illustrato, ci si accorge che il bambino, ancor prima di imparare a leggere, possa essere un lettore: il bambino, quando non è ancora in grado di leggere le parole, legge le immagini. È proprio in età prescolare che si gettano le basi per formare un lettore per tutta la vita. Senza contare le ricadute positive in termini di maturazione di quelle competenze della cosiddetta emergent literacy, ovvero quell'insieme di competenze e di abilità che sono fondamentali per lo sviluppo delle capacità esperte di lettura e scrittura, che emergono tanto più precocemente quanto prima si inizia a leggere. Da qui si afferma che la letteratura per l'infanzia è sempre stata legata alla pedagogia: infatti essa appartiene contemporaneamente a due sistemi, quello educativo e quello letterario, perciò la letteratura infantile non viene esclusivamente letta per l'intrattenimento, ma è anche utilizzata come strumento di educazione e di socializzazione ${ }^{6}$.

L'umanità è ormai da alcuni decenni sommersa dalla rivoluzione digitale cioè dalla diffusione dei cosiddetti digital devices. Tutti i campi della vita quotidiana sono abbondantemente condizionati in modo diretto ossia indiretto

${ }^{6}$ Cfr. per esempio Puurtinen, Tiina, Linguistic acceptability in translated children's literature, n. 15, University of Joensuu, 1995.

Faculty Of Arts Journal 
From Shakespeare to Modern Ages

dall'utilizzo dei dispositivi della nuova media. Oggi è ovviamente innegabile l'incessante espandersi della realtà virtuale nella nostra vita quotidiana. L'avvento e la grande diffusione della Rete ha ampiamente influenzato, e qualche volta alterato, il nostro modo di comunicare, di apprendere alcune realtà e di intendere certe tematiche. Di fronte a questo grande fenomeno, la letteratura non poteva rimanere sorda: non potevano rimanere al riparo la letteratura in generale e la letteratura per l'infanzia in particolare. La letteratura in rete si sviluppa essenzialmente secondo cinque modelli facilmente identificabili: trasposizione di opere cartacee (più o meno famose); prosa; poesia; forme aperte e in progress quali la scrittura collaborativa (moderata e non); e infine forma diaristico-epistolare o Weblog.

Oggigiorno con la società pervasa da dispositivi digitali, la letteratura nell'ambiente digitale è diventata una forma di espressione del nuovo uomo tecnologico. In effetti così come il racconto orale ha condizionato il cantastorie e il libro ha condizionato il romanziere, oggi la tecnologia digitale entra prepotentemente nei processi narrativi dando vita a numerose forme di racconto: letteratura generativa, ipertesto, cybertesto, codework, database fiction, wandering stories, fanfiction, scanfiction, social media storytelling. ${ }^{7}$

La tecnologia digitale ha veramente creato un altro ambiente che può sostituire l'ambiente reale e fornire un nuovo ambiente virtuale, una vera seconda vita che è parallela e adiacente alla nostra esistenza fisica: sotto la spinta della potentissima molla del denaro e del potere che chi fornisce questi prodotti e gestisce questi processi può illimitatamente accumulare nel contemporaneo mondo globalizzato,

7 Cfr. Carbone, Paola, Una brevissima storia della letteratura elettronica, nova100.ilsole24ore.com, 26 ottobre 2011, https://marcominghetti.nova100.ilsole24ore.com/2011/10/26/una-brevissima-storia-dellaletteratura-elettronica-by-paola-carbone-1-generative-literature/ cliccato il 15/7/2020. 
derubandoci del tempo, della cultura, dell'identità, dell'attenzione, della memoria, della realtà, e spingendoci all'auto-confinamento. Nuovi strumenti digitali hanno cambiato il nostro modo di pensare e la struttura del cervello. Isolandoci davanti allo schermo (dove giochiamo, comunichiamo, studiamo, guardiamo film e video, ci informiamo, litighiamo, imitiamo ogni esperienza reale persa nel mondo virtuale, perdiamo il nostro attaccamento reciproco e perdiamo attività, interesse, e d'altra parte, otteniamo uno stereotipo e una rappresentazione standardizzata del mondo. Inganniamo noi stessi e gli altri in modo da avere una migliore comprensione del mondo reale, mentre "ci aggiriamo dappertutto senza fare nessuna esperienza"8.

A tal proposito, occorre innanzitutto porre una netta distinzione tra la letteratura digitale e la letteratura elettronica. La letteratura digitale include qualsiasi testo letterario in formato digitale, e quindi per esempio anche qualsiasi capolavoro della letteratura italiana in formato pdf. La letteratura elettronica, invece, si riferisce a quelle opere concepite per essere, appunto, create e fruite attraverso un medium digitale - laptop, desktops, servers, smartphones, game consoles, interactive environment controllers, - e che sono condizionate dai software o dalle applicazioni con cui sono state create e che ne determinano le modalità di comunicazione: flash, storyspace, html, facebook, email, instagram, twitter, ecc.

Un esempio della letteratura digitale è la letteratura generativa, vale a dire quei testi auto-generati da un algoritmo, preventivamente definito da un programmatore e da un artista, che generalmente viene lanciato dal lettore. Il primo esempio risale alle Loveletters (1952, http: //alpha60.de/research/muc/) del fisico Christopher Stratchey che, sfruttando il Mark I, un computer installato all'università di Manchester, crea un

${ }^{8}$ Byung-Chul, Han, Nello sciame. Visioni del digitale, Nottetempo, Bologna 2016, p. 70. 
From Shakespeare to Modern Ages

programma basato sulla combinatoria che produce lettere d'amore.

Un altro esempio più recente della letteratura digitale è senza dubbio la narrazione nei social media: dal 2005 blogging, photo-blogging, video-blogging hanno contribuito alla trasformazione del consumatore di informazioni in rete in un produttore mediale, basti pensare alle piattaforme di usergenerated-content quali youtube, flicker, myspace. I tag, ossia quelle parole chiave che associate a un contenuto, come un post in un blog, un'immagine, un articolo o un video, permettono di catalogare qualsiasi contenuto, di renderlo rinvenibile e facilmente individuabile dai motori di ricerca. I tag permettono anche di visualizzare graficamente le interconnessioni tra gli oggetti presenti nel web. Gli artisti non potevano affatto ignorare questa nuova opportunità offerta loro dalla tecnologia. Di fatto come un tempo si generavano testi a partire da un algoritmo più o meno sofisticato e ispirato ai principi della combinatoria, oggi si possono sfruttare i flussi ininterrotti di dati provenienti dalla rete facendo leva sul medesimo principio della generazione automatica dei testi. Se da un numero alto ma finito di dati si ricavava un numero ancora più alto di testi, oggi la rete si comporta come un connettore di utenti/nodi di informazioni che possono essere visti come potenziali cyberauthor.

La differenza rispetto ai primi generatori di testi sta nell'utilizzo della network communication per cui è la folla che decide l'instabilità e la mutabilità del testo. In questo nuovo ambito di scrittura, cioè l'ambito tecnologico, l'autore dell'opera fa da ideatore dell'interfaccia, ma ha perso il controllo sui contenuti che vengono determinati dai gusti e dagli interessi del fruitore/utente. Ne consegue che la stessa idea d'interazione, che ha sempre lasciato credere all'intenzionalità nel rapporto tra soggetto e medium digitale, diventa una interazione passiva in cui il fruitore è co-autore in/consapevole 


\section{Dahlia Kashmiry, PhD}

dell'esperienza creativa. Il web 2.0 è geneticamente sociale e serve per condividere esperienze e contenuti. Si consideri per esempio la possibilità di vedere un film in compagnia degli amici di Facebook o l'uso di piattaforme per la creazione (produzione, fruizione e comunicazione) di racconti che includono anche le narrazioni dedicate alle marche di prodotti commerciali (brand storytelling).

È innegabile che le nuove tecnologie rappresentino un'evoluzione nei processi di scrittura, archiviazione, informazione e comunicazione. Scrivere un testo, preparare un compito in classe, formulare un progetto, inviare o chiedere informazioni, materiali o documenti via e-mail, osservare e mostrare immagini e video: questi sono solo alcuni esempi delle tante attività legate al lavoro che le nuove tecnologie hanno reso più agevoli. Senza contare le potenzialità offerte da Internet: trovare rapidamente in rete prove d'esame, riferimenti e bibliografici e sitografici, notizie, articoli, interventi e testi implica indubbiamente il risparmio di un tempo che può essere più proficuamente occupato.

Le nuove tecnologie devono comunque costituire un sostegno anche nell'attività di apprendimento: già sufficientemente abile nel copiare le versioni o nel leggere $\mathrm{i}$ riassunti dei testi online, ogni studente del terzo millennio deve imparare ad accedere criticamente a Internet, deve conoscere i principali motori di ricerca, deve saper maneggiare i più efficaci sistemi applicativi, deve saper individuare e costruire una sitografia attendibile. Su questi aspetti della questione l'attenzione di docenti di tutte le discipline deve essere sempre vigile e costante, perché oggi le aziende e la tecnologia digitale sono diventate dei maestri, mentre il tempo di apprendimento in classe oggi è solo un sostituto dei compiti a casa.

La vita della nuova generazione non è definita a caso la vita dei "nativi digitali" in quanto è permeata dalle nuove 
From Shakespeare to Modern Ages

tecnologie. I computer sono sempre sul tavolo, così come gli schermi dei telefoni cellulari, che possono essere silenziosi ma mai spenti, e lo stesso vale per gli schermi TV in cucina e nel soggiorno di casa. A scuola, e quasi solo a scuola, usano ancora i libri. Smartphone, tablet, personal computer e innumerevoli altri strumenti digitali inondano le nostre case, i luoghi di lavoro, le scuole, le università, le borse e le tasche. Lavoro, acquisto, prenotazione, comunicazione, tempo libero, informazione, controllo, dialogo, intrattenimento: ora tutto avviene principalmente davanti allo schermo, seduto su una sedia, da solo, e irresistibilmente attratto da questa dimensione immateriale.

Le innovazioni introdotte dalle nuove tecnologie hanno causato profondi cambiamenti nelle società, modificando i modi di vivere e di comunicare di una popolazione e rappresentando un punto di svolta culturale per la propria epoca e per le comunità coinvolte. La rapidità della diffusione mondiale dei nuovi dispositivi tecnologici è stata certamente tempestiva e senza precedenti. A tale proposito risulta estremamente significativa l'affermazione di Manuel Castells:

[...] negli Stati Uniti la radio ha impiegato oltre trent'anni per raggiungere sessanta milioni di persone, la televisione ha raggiunto questo livello di diffusione in quindici anni; Internet lo ha fatto in soli tre anni dalla nascita del world wide web ${ }^{9}$.

Pur riferendosi a Internet in modo particolare, l'affermazione di Castell può essere estesa a tutti quei dispositivi tecnologici che generalmente catalogati sotto il nome di ICT (Information and Communication Technology) e che costituiscono il campo dei nuovi media che hanno rivoluzionato l'intero mondo dei mezzi della comunicazione di massa.

\footnotetext{
${ }^{9}$ Castells, Manuel, La nascita della società in rete, Università Bocconi, Milano 1996, p. 382 .
} 


\section{Dahlia Kashmiry, PhD}

Secondo Rodotà la tecnologia "libera la vita da antiche schiavitù, quelle dello spazio e del tempo, e questo è già realtà per milioni di persone"10. In effetti la rete non è soltanto il più grande spazio pubblico che l'umanità abbia conosciuto, anzi è un luogo dove la vita cambia qualità e colore, dove sono possibili l'anonimato e la moltiplicazione delle identità, la conoscenza e l'ubiquità, la libertà piena e il controllo totale. La grande trasformazione tecnologica cambia il quadro dei diritti civili e politici, ridelinea il ruolo dei poteri pubblici, modifica i rapporti personali e sociali, e incide sull'antropologia stessa delle persone. La connessione in rete migliora e velocizza le attività umane in molti ambiti, lavorativi e non, presentando notevoli vantaggi.

Tali vantaggi non si limitano comunque alla sfera degli adulti, in quanto, come s'è detto prima, i dispositivi mobili dalla tecnologia tattile hanno sicuramente rivoluzionato le possibilità di accesso dei bambini piccolissimi alle tecnologie digitali. L'utilizzo del computer richiede certe competenze di base indispensabili per utilizzare in modo adeguato il mouse e la tastiera, ma tablet e smartphone consentono anche ai più piccoli di superare le barriere linguistiche e di interazione delle interfacce precedenti accedendo, in tal modo, direttamente ai contenuti digitali e vivendo esperienze che soltanto alcuni anni fa erano impensabili, come, per esempio, vedere video, giocare, leggere storie. La nascita delle tecnologie touch risale all'inizio degli anni Novanta, ma la vera rivoluzione inizia a partire dal 2007, quando Apple lancia sul mercato la prima versione dell'iPhone. Nel 2010 si diffondono, inoltre, nuovi dispositivi caratterizzati da una diversa interfaccia rispetto ai precedenti, sviluppati esplicitamente per la gestione tramite tocco. Grazie allo schermo tattile viene così ripristinata, nell'interazione con la tecnologia, la corporeità e ciò permette anche ai bambini più

10 Rodotà, Stefano, Persona, libertà, tecnologia. Note per una discussione, Diritto\&Questioni Pubbliche, n. 5, 2005, p. 25.

Faculty Of Arts Journal 
From Shakespeare to Modern Ages

piccoli, che non sanno leggere e scrivere e non hanno raggiunto determinati livelli rispetto alla coordinazione oculo-manuale, di interagire con le tecnologie molto prima rispetto al passato, superando così barriere che per molto tempo hanno impedito di accedere ai nuovi media e fruire delle loro potenzialità.

Al riguardo degli effetti che le tecnologie digitali possono produrre sugli esseri umani, in particolare sui bambini, ci sono infatti varie posizioni. Dopo i primi anni, segnati da un acritico entusiasmo e celebrazione delle opportunità cognitive, didattiche e partecipative presentate da Internet, sono sorte tante posizioni contro le tecnologie digitali, con particolare attenzione agli effetti negativi che queste possono avere sui bambini ${ }^{11}$. Bisogna ricordare innanzitutto che un gran numero di pubblicazioni si schierano senza tanti preamboli contro l'uso delle ICT. Si tratta dei cosiddetti tecnofobi, che ribadiscono che tecnologie digitali, internet e social network arrecano danni irreparabili all'umanità e, in particolare, alle nuove generazioni e suggeriscono di evitare o almeno di ritardare il più possibile il contatto con i bambini.

Gli strumenti digitali a noi sempre e ovunque disponibili vengono creati da aziende che mirano a fare profitto; che appartengono a una rete che registra i nostri gusti tramite le informazioni da noi stessi fornite, inconsciamente o meno; i prodotti che ci vengono offerti appartengono così a una stretta minoranza ricorrente di oggetti di consumo, che immobilizzano le nostre possibilità di scelta, pur nell'apparente vastità dell'offerta. Allo stesso modo il libro di carta è soggetto a questi rischi, ma i vantaggi cognitivi e sociali - secondo Roberto Casati - offrono valide argomentazioni per la sua difesa e

\footnotetext{
11 A tal proposito si possono consultare Turkle, Sherry, Insieme ma soli. Perché ci aspettiamo sempre più dalla tecnologia e sempre meno dagli altri, Codice Edizioni, Torino 2012; Morozov, Evgenij, L'ingenuità della rete: il lato oscuro della libertà di internet, Codice Edizioni, Torino 2011; Lovink, Geert, Ossessioni collettive. Critica dei social media, EGEA-Università Bocconi, Milano 2012.
} 
proposte di discussione per chi fa formazione ai tempi del digitale, anche all'università. Secondo Casati non ci sono dei 'nativi digitali', al limite dei 'nati digitali'. Bisogna evitare l'idea che l'assuefazione ai nuovi media digitali sia scontata e produca automaticamente competenze; bisogna prendere con estrema cautela l'idea di un nuovo modello antropologico inevitabile. Le nuove generazioni esposte al digitale non sono né malati né mutanti ${ }^{12}$.

È ormai chiaro che, a seguito del nuovo ambiente digitale nel quale sono immersi e della crescita esponenziale della loro interazione con gli strumenti della rivoluzione digitale, i bambini e gli studenti di oggi apprendono e gestiscono l'informazione e la comunicazione in modo sostanzialmente diverso da noi, loro predecessori ${ }^{13}$.

Altra posizione decisamente negativa nei confronti dei nuovi media è quella adottata dallo psichiatra Manfred Spitzer che analizza in due libri ${ }^{14} \mathrm{i}$ rischi provocati dalla digitalizzazione. Egli ritiene che la tecnologia digitale non ci rende soltanto stupidi, ma addirittura dementi. Il suo avvertimento concerne i pericoli che corre il cervello umano costantemente a contatto con il digitale. L'espressione demenza digitale si riferisce alla sindrome individuata nel 2008 da alcuni specialisti in Corea del Sud, paese ad alta diffusione di tecnologia informatica, che colpiva i giovani dediti all'uso dei media digitali per molte ore al giorno e caratterizzata da appiattimento emotivo, difficoltà di attenzione e di memoria e un generale declino delle capacità di apprendimento. L'utilizzo costante dei media digitali, secondo Spitzer, ha un effetto negativo sullo sviluppo cognitivo degli esseri umani: tale effetto

\footnotetext{
${ }^{12}$ Cfr. Casati, Roberto, Contro il colonialismo digitale. Istruzioni per continuare a leggere libri, Laterza, Roma-Bari 2013, p. 95.

${ }^{13}$ Ferri, Paolo, Nativi digitali, Bruno Mondadori, Milano 2011, p. 12.

${ }^{14}$ Questi due libri sono Spitzer, Manfred, Demenza digitale. Come la nuova tecnologia ci rende stupidi, Corbaccio, Milano 2013; e Spitzer, Manfred, Solitudine digitale, Disadattati, isolati, capaci solo di una vita virtuale?, Tr. it., Corbaccio, Milapo 2016 
From Shakespeare to Modern Ages

consiste in una riduzione del tempo di elaborazione degli stimoli necessario affinché si verifichi un buon apprendimento (in particolare attraverso il sostare attivo dell'attenzione su uno stesso stimolo) e dalla facile distrazione coesistente cui l'individuo è indotto dall'interferenza continua dei molteplici elementi presenti su uno schermo (cosa che induce al cosiddetto multitasking, ossia al balzare dell'attenzione celermente da uno stimolo all'altro). Sono effetti che, con il passare del tempo, potrebbero modificare il funzionamento dei processi coscienti e di autocontrollo cognitivo.

Un altro aspetto molto influenzato dalla diffusione della tecnologia digitale è senz'altro la presenza del libro e della lettura nella nostra vita quotidiana, sia a livello puramente dilettevole che a livello istruttivo e didattico. Oltre all'intrattenimento, una delle funzioni della letteratura è sempre stata quella di mediare il rapporto tra la persona e la sua contemporaneità e di renderlo comprensibile, oppure di rendere i lettori consapevoli della complessità della realtà attraverso la narrazione della storia. Oggi si legge sempre di meno. In Italia, una fra le G7 del mondo, più del $35 \%$ della popolazione, secondo i dati pubblicati dall'Associazione Italiana Editori ${ }^{15}$ per l'anno 2019, non legge neanche un libro in tutto l'anno. Si tratta di un'analisi impietosa, ma utile, di una dimensione molto discussa da tutti, ma nell'agenda di quasi nessuno: la cultura. Per leggere qui, si intendono non solo i libri cartacei ma tutti i tipi dei libri, sia cartacei che ebook che audiolibri, e non solo libri di letteratura o di narrativa ma libri di tutti i tipi e generi (come un romanzo, un giallo, fantasy, un graphic novel, ecc.), ma anche un saggio, un manuale, una guida di viaggio o di cucina, sulla salute, ecc.) Secondo i dati dell'AIE, dei libri letti nell'ultimo anno, il $62 \%$ sono dei libri cartacei, il $25 \%$ sono ebook e il $9 \%$ sono audiolibri. Per quanto riguarda le fasce di

${ }^{15}$ www.aie.it , cliccato il 20/5/2020. 


\section{Dahlia Kashmiry, PhD}

età dei lettori, si nota che i giovani leggono più degli adulti: i lettori fra 6-19 anni costituiscono il 53.1\% mentre quelli fra 4574 anni raggiungono solo il 38,4\% del totale dei lettori. Per quanto riguarda $\mathrm{i}$ generi favoriti dai lettori italiani, si deduce dai dati dell'AIE che il 25.4\% dei libri letti nel 2018 sono libri di narrativa italiana, il $39 \%$ sono libri per bambini e ragazzi, il $20.5 \%$ libri di saggistica, e il $10.7 \%$ sono libri illustrati.

Il rapporto tra bambino e libro, oggi, diviene ulteriormente problematico per effetto dell'ampia diffusione delle nuove tecnologie. È vero che i bambini, oggi, sanno maneggiare sin da piccolissimi gli strumenti elettronici e sono grandi consumatori di cartoni animati e di applicazioni per smartphone e tablet. Si tratta di forme di narrazione che li abituano a una modalità di fruizione delle storie molto più veloce rispetto a quella offerta dal classico libro letto dai genitori. Il bambino si abitua così ad una rapida serie di emozioni che lo condizioneranno anche quando imparerà a soddisfare la sua passione di storie attraverso le pagine di un libro letto direttamente. Si verifica purtroppo una malintesa accezione di rapidità che si traduce così nell'offerta, da parte di numerose case editrici, di storie, rivolte anche alla fascia dei piccolissimi (0-6), dal taglio narrativo esile e dall'apparato iconografico stereotipato, per lo più dominate dal topino, principessa di turno, da ritrovare puntualmente su zainetti, quaderni, diari e una serie infinita di gadget. In fin di conti rimangono storie che non si accumulano nell'animo del bambino che le legge e che sono come acqua che scorre sopra il letto di un fiume. Storie che, sostiene Giorgia Grilli, vedono l'infanzia come un'età imperfetta piuttosto che come una dimensione in cui si sperimenta una fondamentale alternativa esistenziale e la letteratura per l'infanzia come una letteratura di 
From Shakespeare to Modern Ages

nessun conto, poco degna di attenzione anziché come discorso ricco di imprescindibili spunti di riflessione ${ }^{16}$.

Da una ricerca on-line curata da Nati per Leggere ${ }^{17}$ (marzo 2013) sul rapporto tra famiglie e libri digitali per l'infanzia in Italia, con maggiore attenzione anche al mondo della scuola e delle biblioteche, emerge che solo il $30 \%$ dei genitori partecipanti alla ricerca usa abitualmente i libri digitali con i propri bambini, mentre più del $60 \%$ preferisce la lettura del libro di carta: i libri digitali generalmente vengono usati quando si è in viaggio, in auto, o nei momenti di intrattenimento, mentre per scandire i momenti rituali, quali, ad esempio, il sonno, si preferisce il libro cartaceo. Si attesta intorno al $70 \%$ anche il numero degli insegnanti e dei bibliotecari che dichiarano di non usare in alcun modo i media digitali, generalmente perché non in possesso di strumenti idonei per leggerli. Il libro digitale può infatti sottrarre magia alla lettura e momenti di condivisione adulto-bambino, ed è un fatto che diviene ancora più comprensibile se si pensa che nella maggioranza dei casi (il 70\%) le famiglie italiane impiegano il libro come momento di accompagnamento al sonno. Altre spiegazioni interessanti, il libro digitale può far perdere il rapporto sensoriale con il libro e creare eccessiva dipendenza dal supporto digitale, disabituare ai ritmi lenti tipici della lettura del libro di carta e ridurre la capacità di mettere in sequenza logico-temporale le fasi di una storia. Una diffidenza che non appartiene a quella percentuale che invece conosce ed utilizza anche e-book ed app.

\footnotetext{
${ }^{16}$ Cfr. Grilli, Giorgia, Bambini, insetti, fate e Charles Darwin. In Beseghi, Emy \& Grilli, Giorgia (a cura di). La letteratura invisibile (pp. 21-57), Carocci, Roma 2011, p. 57.

17 Cfr. http://www.natiperleggere.it/wp/wp-content/uploads/2019/10/Report-20anni_COMPLETO-WEB-protetto.pdf . Il questionario, strumento di raccolta dei dati della ricerca, è stato lanciato nel mese di gennaio da Filastrocche.it, Happi ideas, Mamamò e Nati per Leggere, in collaborazione con AIB (Associazione Italiana Biblioteche), MLOL (MediaLibraryOnLine) e FattoreMamma.
} 


\section{Dahlia Kashmiry, PhD}

Al di là delle idee degli adulti, tuttavia, è certo che $\mathrm{i}$ bambini e gli adolescenti italiani abbiano un rapporto sempre più pervasivo e preminente con le nuove tecnologie. Un dato che non va visto necessariamente in chiave negativa rispetto al coinvolgimento nella lettura, poiché l'uso delle nuove tecnologie non impedisce di essere lettori forti e i bambini e i ragazzi lettori si approcciano alle nuove tecnologie, audiovisive e digitali, in modo molto più critico e selettivo rispetto ai loro compagni non-lettori. Ciò testimonia che non è più possibile pensare a una cultura dell'infanzia che non comprenda anche il mondo del web e della multimedialità. Uno studio condotto dalla casa editrice Piemme sui propri titoli nel 2011 documenta come i bambini, a cominciare dalla primissima infanzia, privilegino le applicazioni, perché offrono un'esperienza dinamica e interattiva, e quindi più attrattiva e coinvolgente.

Un altro aspetto della rivoluzione digitale che invade la nostra vita quotidiana e in modo particolare la vita dei ragazzi e dei giovani sono i videogiochi. L'atteggiamento nei confronti dei videogiochi è spesso guidato da un pregiudizio negativo, basato su persuasioni ideologiche che li ritengono come fattori negativi d'apprendimento, pur senza una vera base scientifica. In realtà, la letteratura psicologica sottolinea spesso il ruolo positivo dei videogiochi nello stimolare particolari capacità del bambino. I dati dell'Istituto nazionale di statistica ${ }^{18}$ sono interessanti al riguardo e mostrano un andamento discorde nella popolazione tra i 6 e gli 11 anni e in quella di età superiore. È possibile che l'uso dei videogiochi svolga un ruolo pedagogico nell'infanzia e che in età adolescenziale, invece, le consolle si trasformino più facilmente in una fonte di distrazione, per la maggiore autonomia dei ragazzi nel loro utilizzo.

Un altro dato interessante riguarda l'accesso ai social network e ai cellulari, che appare negativamente correlato alla lettura solo per i bambini più piccoli (tra i 6 e gli 11 anni); la

${ }^{18} \mathrm{Cfr}$ http://www.istat.it/it/files/2011/12/ICT-famiglie-2011.pdf 
From Shakespeare to Modern Ages

correlazione diventa invece positiva per gli altri minori. Probabilmente qui viene indirettamente coinvolto il modello educativo dei genitori: l'accesso allo smartphone o l'esistenza di un profilo attivo su un social network per $\mathrm{i}$ bambini più piccoli sarebbe da incrociare con la presenza e il controllo dei genitori, con forte valore pedagogico. Man mano che i ragazzi crescono, gli strumenti tecnologici diventano, invece, occasione di contaminazione e condivisione, spesso funzionale alla lettura o alla scoperta.

Un aspetto molto significativo della rivoluzione digitale è senz'altro l'uso eccessivo e la dipendenza dagli smartphone. Prendendo sempre in considerazione la situazione in Italia, e secondo le rilevazioni Eurispes (2016), lo smartphone si conferma lo strumento tecnologico più diffuso nel Paese: ne possiede uno il 75,7\% degli italiani (era il 67\% nel 2015). L'utilizzo più frequente dichiarato resta chiamare ed essere chiamati (99,3\%), seguono inviare e ricevere sms $(85,1 \%)$. Moltissimi comunicano tramite WhatsApp o altre applicazioni di messaggistica $(75,2 \%)$, fanno foto e filmati (69\%), li inviano e ricevono (68\%), navigano su Internet (66,8\%). La maggioranza usa le applicazioni $(54,2 \%)$ e i Social Network $(51,1 \%)$. Il 43,3\% del campione dichiara inoltre di possedere tablet/ipad, dato in crescita rispetto al 36,8\% dell'anno precedente. Navigare su Internet è ormai un dato di fatto per la maggior parte della popolazione $(81,5 \%)$. Gli italiani usano la Rete soprattutto per cercare informazioni di loro interesse $(97,8 \%)$ e inviare e ricevere e-mail $(85,8 \%)$; per navigare sui Social Network $(68,9 \%)$, guardare filmati su YouTube $(66,8 \%)$, controllare il proprio conto bancario $(65,1 \%)$, fare acquisti $(55 \%)$.

Secondo i dati dell'Istat, l'unico dato costante per ogni fascia d'età, all'interno della popolazione italiana 6-18 anni, riguarda le ore giornaliere passate davanti alla televisione. La correlazione negativa con la lettura è facilmente spiegabile: 


\section{Dahlia Kashmiry, PhD}

davanti alla tv l'atteggiamento è più passivo. Ci sarebbe, probabilmente, da invertire la tendenza, se si considera che in Italia il tempo medio passato davanti alla tv è in costante aumento anche per i bambini più piccoli. Tuttavia, è difficile sostenere che la soluzione al problema dei pochi libri letti in Italia possa risiedere nella compressione dello spazio di cui godono televisione e social network.

Con i dati proposti sulla situazione della lettura e dei digital media in Italia, si vuole asserire che la giovinezza sembra caratterizzarsi come l'età d'oro della lettura; ci sono ancora molti ragazzi e giovani che leggono ancora e soprattutto che le modalità di lettura non sono più come una volta. Anche se i libri tradizionali continuano a resistere, ormai siamo nell'era degli ebook, cioè dei libri elettronici, e degli audiolibri. Negli ambienti educativi e formativi in tempi recentissimi si è diffuso un crescente dibattito in merito a come e se tali dispositivi possono/devono entrare a far parte anche della vita dei bambini, sebbene l'uso di smartphone e tablet, nel frattempo, sia già diventato una realtà per molti di loro.

Serge Tisseron, psicoanalista e psichiatra francese, espone i vantaggi e gli svantaggi derivanti dall'uso della tecnologia digitale, e dedica una riflessione a un tema molto discusso, ovvero il rapporto tra libro (o cultura) di carta e libro (o cultura) digitale. Secondo l'autore l'avvento degli schermi e, di conseguenza, della cultura digitale non ha eliminato e non eliminerà la cultura del libro, poiché

Ciascuna delle due si richiama a un funzionamento cerebrale e psichico differente, in modo tale che l'essere umano va più veloce se le usa tutte e due, esattamente come si muove più rapidamente se utilizza tutte e due le gambe di cui è dotato. ${ }^{19}$ Egli ritiene vano il dibattito che contrappone la creazione digitale al libro e alla scrittura, poiché la realtà "è più

\footnotetext{
${ }^{19}$ Tisseron, Serge, 3-6-9-12. Diventare grandi all'epoca degli schermi digitali, La Scuola, Brescia 2013, p. 106
} 
From Shakespeare to Modern Ages

complessa, ma anche ben più esaltante" ${ }^{20}$. La cultura del libro e quella dei touch screen si contrappongono da tutti i punti di vista e, in particolare, rispetto alla relazione con i saperi e gli apprendimenti, al funzionamento psichico e alla creazione di legami. Egli, tuttavia, partendo proprio dalle differenze che le separano, ribadisce l'indispensabile complementarità delle due culture, senza la quale "ciascuna delle due, se non viene equilibrata dall'altra, comporta pericoli significativi",21. Inoltre Tisseron presenta delle raccomandazioni al riguardo all'educazione dei bambini all'utilizzo del digitale, la quale dovrebbe basarsi, in particolare, su tre considerazioni principali: l'autoregolazione, che il bambino può raggiungere se gli vengono forniti gli strumenti adatti affinché possa scegliere da solo in maniera equilibrata; 1'alternanza, basata "sulla diversificazione degli stimoli e l'incoraggiamento del bambino a sviluppare attività che servano a mobilitare i suoi cinque sensi e le sue dieci dita"22. l'accompagnamento, ovvero la disponibilità dell'adulto ad appoggiare il bambino facendogli raccontare le sue esperienze con gli schermi, in modo che possa utilizzare alternativamente la sua intelligenza spaziale, tipica degli schermi, e quella narrativa, lineare, tipica del linguaggio parlato e scritto.

Del resto, Tisseron, trattando tali raccomandazioni, dedica maggiore attenzione alla fascia dei bambini sotto i tre anni. Egli sottolinea che gli schermi interattivi sembrano bene adattarsi alle forme senso-motorie dell'intelligenza infantile, ma non sono ancora stati valutati gli effetti a lungo termine derivanti dal loro uso. Comprovati da numerosi studi, sono, invece, certi i danni provocati dagli schermi non interattivi (televisione e lettori di dvd): rallentamento degli apprendimenti (in particolare del linguaggio), aumento di peso, incapacità di

\footnotetext{
${ }^{20}$ ivi, p. 95 .

${ }^{21}$ ivi, p. 106.

22 ivi, p. 24.
} 
concentrazione, difficoltà nella socializzazione. Di conseguenza lo studioso consiglia di evitare il più possibile televisione e lettori dvd e, sottolineando che i bambini fanno ricorso all'imitazione fin dalla nascita, suggerisce agli adulti di limitarne l'uso in loro presenza. Una volta si decida di consentire loro di usare tablet o smartphone, bisogna accompagnarli, ridurre $\mathrm{i}$ tempi di utilizzo, scegliere programmi adatti alle loro capacità, senza dimenticare di dedicarsi con loro anche ai giochi tradizionali. Secondo lo studioso non bisogna avere fretta, dal momento che, a suo avviso, i bambini che non hanno un tablet a disposizione non sviluppano alcun ritardo.

Nel 2018 la Società Italiana di Pediatria ${ }^{23}$, nel suo primo documento ufficiale sull'uso di smartphone e tablet da parte dei bambini da 0 a 8 anni di età, sottolinea, accanto ad alcuni vantaggi, i rischi documentati di una esposizione precoce e prolungata ai dispositivi dallo schermo tattile e suggerisce di porre limiti e trovare alternative per intrattenere e calmare i bambini. Facendo riferimento a diversi studi, i pediatri italiani sottolineano le diverse prove scientifiche sulle interazioni delle tecnologie digitali con lo sviluppo neuro-cognitivo, l'apprendimento, il sonno, la vista, l'udito, le funzioni metaboliche. Nel documento si ribadisce che le modalità d'uso di smartphone e tablet da parte dei genitori influenzino la sensazione di sicurezza dei bambini, il loro benessere emotivo e le interazioni tra i membri della famiglia. I digital devices, infatti, distraggono la coppia genitore-figlio dalle interazioni faccia-a-faccia e hanno un grande effetto sullo sviluppo cognitivo, linguistico ed emotivo. Inoltre viene sconsigliata la consuetudine di molti genitori di utilizzare $\mathrm{i}$ dispositivi mobili come strumenti per calmare, distrarre i bambini o controllare il loro comportamento, perché ritenuta negativa per lo sviluppo

${ }^{23}$ Cfr. Bozzola Elena, Spina Giulia, Ruggiero Margherita, Memo Luigi, Agostiniani Rino, Bozzola Mauro, Corsello Giovanni \& Villani Alberto, Media devices in pre-school children: the recommendations of the Italian pediatric society, Italian journal of pediatrics, 44(1), 69, 2018.

Faculty Of Arts Journal 
From Shakespeare to Modern Ages

della regolazione emotiva. I pediatri italiani consigliano che l'esposizione ai touch screen debba essere regolata sulla base delle evidenze cliniche $e$, in particolare, che sia vietata ai bambini al di sotto dei 2 anni di età, durante i pasti, almeno un'ora prima di andare a dormire, di app con contenuti violenti, per mantenere tranquilli i bambini.

Suggeriscono anche di limitare l'esposizione ai media a meno di un'ora al giorno ai bambini di età compresa fra 2 e 5 anni, a meno di due ore al giorno ai bambini tra 5 e 8 anni, di scegliere programmi di alta qualità, di testarli prima che il bambino li utilizzi e di fare in modo che ciò accada solo in presenza degli adulti in modo da incoraggiare gli apprendimenti e le relazioni. Il documento ribadisce, infine che, in quanto i bambini tendono ad imitare gli adulti, i genitori devono limitare l'utilizzo personale dei media e privilegiare situazioni d'interazione diretta con i propri figli che non prevedano l'uso di dispositivi touch.

Come si evince da quanto fin qui esposto, il dibattito intorno ai reali vantaggi e svantaggi derivanti dall'uso dei dispositivi touch da parte dei bambini e alle ricadute sul loro sviluppo risulta essere del tutto aperto. La complessità del fenomeno chiede al mondo della ricerca di descrivere, in modo cauto e rigoroso, quanto e come gli strumenti digitali siano presenti nella vita e quali siano i fattori legati all'uso da parte loro di tali strumenti che meglio supportano oppure ostacolano il loro sviluppo e i loro processi di apprendimento.

Con tutti questi vantaggi e svantaggi della diffusione dei digital devices, dobbiamo ammettere che qualsiasi tentativo di pretendere di rubare spazio a questi mezzi equivalga a cercare di impedire a un fiume di fluire verso il mare: non è possibile combatterli perché significherebbe negare l'essenza della cultura contemporanea. Certo, ognuno di noi, sulla base della propria consapevolezza, ne può regolare l'uso con intelligenza. Ma si tratta appunto di azioni affidate al singolo e alla sua 


\section{Dahlia Kashmiry, PhD}

crescita personale. Non è più possibile, d'altra parte, pensare a una cultura dell'infanzia che non comprenda anche il mondo del web e della multimedialità. In Italia una recente indagine di Bruschi e Carbotti ${ }^{24}$, condotta secondo una prospettiva pedagogica, si è parlato di potenzialità e criticità dei dispositivi mobili, la cui diffusione ha portato a sistema il principio della trasportabilità e ha introdotto il meccanismo delle app. Le autrici evidenziano innanzitutto i rischi relativi al fatto che ogni giorno vengono immesse sul mercato decine di applicazioni rivolte a tutte le tipologie di utenti, che stimolano i possessori di tecnologie touch a comprare, scaricare, consumare senza valutarne la qualità. Questo fenomeno è particolarmente critico al riguardo dei prodotti rivolti ai più piccoli. Le studiose pongono l'attenzione sui criteri che consentono di progettare e scegliere una buona app per bambini e, tra $\mathrm{i}$ numerosi aspetti presi in considerazione, analizzano in particolare l'uso dei personaggi, l'interattività, il design (che comprende l'uso di metafore, forme, colori, icone), il testo, la navigazione e l'audio (speakeraggio, musica, effetti sonori).

In conclusione alla presente analisi, va sottolineato che l'apprendimento della letteratura in generale e della letteratura per l'infanzia in particolare non può essere più attuato solo attraverso i metodi tradizionali, e che esso deve adattarsi alla nuova realtà digitalizzata. Di conseguenza, la scuola, l'università e tutte le istituzioni pedagogiche, volenti o nolenti, devono fare i conti con la rivoluzione digitale, che ha modificato assai l'immaginario e l'esperienza del mondo. Il fenomeno è di tali dimensioni sul piano antropologico e culturale, che non è più pensabile continuare a proporre la letteratura con le stesse modalità sperimentate con successo prima dell'avvento del web, dei social network, dei motori di ricerca.

\footnotetext{
${ }^{24}$ Cfr. Bruschi, Barbara \& Carbotti, Silvia, Per imparare c'è un'qun,_ Aracne, Roma 2012 


\section{From Shakespeare to Modern Ages}

Oggi le tecnologie dell'informazione e della comunicazione non solo hanno invaso la vita quotidiana, soprattutto delle giovani generazioni, ma sono entrate anche nell'ambiente scolastico; la loro presenza all'interno delle aule e tra $\mathrm{i}$ banchi di scuola è destinata a crescere e per questa ragione i docenti dovrebbero essere in grado di riflettere sul significato del cambiamento in corso e individuare strategie per accompagnarlo. Il legame tra le tecnologie, la letteratura e la didattica deve necessariamente essere affrontato, studiato e sperimentato nelle scuole, con lo scopo di alimentare un dibattito pubblico ancora immaturo, che necessita di un approccio meno ideologico e più realista.

Numerose ricerche ${ }^{25}$ hanno dimostrato che un incontro con libri e lettura precoce, positivo e che coinvolge tutti i sensi ha importanti implicazioni con la nascita e lo sviluppo dell'amore per libri e lettura e per lo sviluppo cognitivo, emotivo e sociale dei bambini. Risulta quindi urgente studiare e comprendere, attraverso un metodo colloquiante e partecipativo, che si ponga al crocevia di diversi ambiti disciplinari, l'incontro, e non lo scontro, tra il libro, gli altri media e i bambini, per comprendere se e come le nuove tecnologie cambieranno il modo in cui si legge e ci si appassiona alla lettura.

Un altro tema intorno al quale si discute sempre più spesso e che deve essere attentamente indagato con rigore e cautela è sicuramente quello riguardante la «dieta digitale» più equilibrata con cui «nutrire» i nativi digitali, ovvero la qualità e la quantità dei «cibi mediatici» che vengono offerti ai più piccoli, in modo da costruire «mappe ragionate» dei contenuti che possono essere più o meno adeguati a un così particolare tipo di utenti e da fornire indicazioni supportate dalle

\footnotetext{
${ }^{25}$ Cfr per esempio Detti, Ermanno, Il piacere di leggere, La Nuova Italia, Firenze 1987; e Blezza Picherle, Silvia, Libri, bambini, ragazzi. Incontri tra educazione e letteratura, Vita e Pensiero, Milano 2004.
} 


\section{Dahlia Kashmiry, PhD}

evidenze della ricerca sulla «giusta misura» del tempo che può essere trascorso davanti agli schermi, allo scopo di supportare genitori, educatori e insegnanti nel ruolo di accompagnatori nel mondo digitale ${ }^{26}$.

Sulla base di questa prospettiva, diviene indispensabile che i servizi educativi per l'infanzia non rinuncino al loro importante ruolo di laboratori naturali di ricerca e monitoraggio dell'esperienza della crescita, per diventare ambienti in cui i bambini possano agire come costruttori del proprio sapere e fare esperienze che impegnino la mente e il corpo e che permettano loro di mettere da parte un'idea di tecnologia che isola, anestetizza ed accentra a favore di un'idea di tecnologia che arricchisce e consolida i modi di conoscere.

Si augura che il mondo della ricerca lavori al fianco di pedagoghi ed insegnanti contribuendo in tal modo a valorizzare procedure riflessive relative all'azione che possano contribuire alla definizione di strategie consapevoli e capaci di rispondere in modo adeguato alle sfide che le tecnologie digitali pongono all'educazione. La definizione di buone pratiche e la disponibilità di buoni prodotti contribuiranno a modificare in modo determinante la qualità delle esperienze e il sistema di interazioni didattiche, a favore di un uso consapevole e attivo delle tecnologie touch da parte dei giovani, dei ragazzi e in particolare dei bambini.

\footnotetext{
${ }^{26}$ Ripamonti Donata, Bambini e tecnologie digitali: opportunità, rischi e prospettive di ricerca, Media Education - Studi, ricerche, buone pratiche Vol. 7, n. 2, (pp. 143-157), Edizioni Centro Studi Erickson S.p.a., 2016, p. 154.
} 
From Shakespeare to Modern Ages

\section{Bibliografia}

- Angelucci, Anna, I giovani, il digitale e il cambiamento mentale, 12 Giugno 2017, La letteratura e noi, diretto da Romano Luperini https://www.laletteraturaenoi.it/.

- Angelucci, Anna, Sull'utilità dell'applicazione delle nuove tecnologie alla didattica della letteratura, 22 Marzo 2013, La letteratura e noi, diretto da Romano Luperini https://www.laletteraturaenoi.it/.

- Balboni, Paolo E. (a cura di), Educazione letteraria e nuove tecnologie, Utet Libreria, Torino 2004.

- Beckett, Sandra, Crossover fiction: global and historical perspectives, Children's literature and culture, Routledge, New York 2009.

- Blezza Picherle, Silvia, Libri, bambini, ragazzi. Incontri tra educazione e letteratura, Vita e Pensiero, Milano 2004.

- Bozzola Elena, Spina Giulia, Ruggiero Margherita, Memo Luigi, Agostiniani Rino, Bozzola Mauro, Corsello Giovanni \& Villani Alberto, Media devices in pre-school children: the recommendations of the Italian pediatric society, Italian journal of pediatrics, 44(1), 69, 2018.

- Bruschi, Barbara \& Carbotti, Silvia, Per imparare c'è un'app, Aracne, Roma 2012.

- Byung-Chul, Rodotà, Stefano, Persona, libertà, tecnologia. Note per una discussione, Diritto\&Questioni Pubbliche, n. 5, 2005 Han, Nello sciame. Visioni del digitale, Nottetempo, Bologna 2016.

- Campagnaro, Marnie, Narrare per immagini. Uno strumento per l'indagine critica, Collana $i$ Saperi della Formazione, Pensa Multimedia, Lecce 2012. 
Dahlia Kashmiry, PhD

- Carbone, Paola, Una brevissima storia della letteratura elettronica, nova100.ilsole24ore.com, 26 ottobre 2011, https://marcominghetti.nova100.ilsole24ore.com/2011/10 /26/una-brevissima-storia-della-letteratura-elettronica-bypaola-carbone-1-generative-literature/.

- Casati, Roberto, Contro il colonialismo digitale. Istruzioni per continuare a leggere libri, Laterza, RomaBari 2013.

- Caso, Rossella, Dal libro di carta alla app_le nuove frontiere della narrazione in età prescolare, Metis Mondi educativi. Temi indagini suggestioni, Anno III Numero 1 - 06/2013.

- Castells, Manuel, La nascita della società in rete, Università Bocconi, Milano 1996.

- Detti, Ermanno, Il piacere di leggere, La Nuova Italia, Firenze 1987.

- Di Lella, Alessio, La scrittura creativa alla prova del cyberspazio, in AA. VV., La letteratura nell'era dell'informatica - proposte per il XXI secolo, Bevivino Editore, Milano 2007-2008.

- Ferri, Paolo, Nativi digitali, Bruno Mondadori, Milano 2011.

- Grenby, Matthew O. e Reynolds Kimberely (ed.), Children's Literature Studies: a Research Handbook, Palgrave Macmillan. 4, Basingstoke 2011.

- Grilli, Giorgia, Bambini, insetti, fate e Charles Darwin. In Beseghi, Emy \& Grilli, Giorgia (a cura di). La letteratura invisibile (pp. 21-57), Carocci, Roma 2011.

- La Marca, Alessandra, Educazione del carattere, letteratura per l'infanzia e stile di vita digitale, Un percorso di formazione iniziale degli insegnanti, Giornale italiano della ricerca educativa, 10, 367-383, 2017.

- Lovink, Geert, Ossessioni collettive. Critica dei social media, EGEA-Università Bocconi, Milano 2012. 
From Shakespeare to Modern Ages

- Morozov, Evgenij, L'ingenuità della rete: il lato oscuro della libertà di internet, Codice Edizioni, Torino 2011.

- Nodelman Perry, Illustration and Picture Books. in Hunt, Peter, "Children's Literature". In P. Nel e L. Paul (ed.). Keywords for Children's Literature, New York University Press, New York 2011.

- Nodelman, Perry, The pleasures of children's literature, Longman, New York 1992.

- Puurtinen, Tiina, Linguistic acceptability in translated children's literature, n. 15, University of Joensuu, 1995.

- Reynolds, Kimberely, Children's Literature: A Very Short Introduction, Oxford University Press, Oxford 2011.

- Ripamonti, Donata, Bambini e tecnologie digitali: opportunità, rischi e prospettive di ricerca, Media Education - Studi, ricerche, buone pratiche Vol. 7, n. 2, (pp. 143-157), Edizioni Centro Studi Erickson S.p.a., 2016.

- Riva, Francesca, Insegnare letteratura nell'era digitale, Edizioni ETS, Pisa 2017.

- Ruggiero, Alessandra (et al.), Culture in transito, Letteratura, media e tecnologie digitali, Between, vol. IV, n. 8 (Novembre 2014).

- Spitzer, Manfred, Demenza digitale. Come la nuova tecnologia ci rende stupidi, Corbaccio, Milano 2013.

- Spitzer, Manfred, Solitudine digitale, Disadattati, isolati, capaci solo di una vita virtuale?, Tr. it., Corbaccio, Milano 2016.

- Tisseron, Serge, 3-6-9-12. Diventare grandi all'epoca degli schermi digitali, La Scuola, Brescia 2013.

- Turkle, Sherry, Insieme ma soli. Perché ci aspettiamo sempre più dalla tecnologia e sempre meno dagli altri, Codice Edizioni, Torino 2012. 


\section{$\underline{\text { Sitografia }}$}

- wWW.aie.it

- www.natiperleggere.it

- www.istat.it 